\title{
Inaccessible cardinals without the axiom of choice
}

\author{
by
}

\author{
Andreas Blass (Ann Arbor, MI), Ioanna M. Dimitriou (Bonn) \\ and Benedikt Löwe (Amsterdam, Bonn and Hamburg)
}

\begin{abstract}
We consider four notions of strong inaccessibility that are equivalent in ZFC and show that they are not equivalent in ZF.

1. Introduction. Large cardinals serve as a calibration of logical strength for axiom systems of set theory. If we compare theories up to equiconsistency, every natural set-theoretic axiom system is equiconsistent with some $Z F C+L$ where $\mathrm{L}$ is a large cardinal axiom. These axioms come in many guises, and most of the large cardinal axioms have several equivalent definitions. For instance, a cardinal $\kappa$ is measurable if there is a $\kappa$ complete nonprincipal ultrafilter on it, but in the presence of the axiom of choice you can show that this is equivalent to the statement "there is an elementary embedding of the universe into a transitive class with critical point $\kappa "$.

If you move into the choiceless world, some of these ZFC-equivalent definitions are not equivalent anymore. The cardinal $\aleph_{1}$ can never be the critical point of an elementary embedding of the universe into a transitive class, but it is possible that it carries a $\sigma$-complete nonprincipal ultrafilter in models of ZF $\left.{ }^{1}\right)$. It is interesting to analyze how much of the robustness of large cardinal notions we lose if we give up the axiom of choice.
\end{abstract}

2000 Mathematics Subject Classification: Primary 03E10, 03E25; Secondary 03E55.

Key words and phrases: inaccessible cardinal, axiom of choice.

$\left({ }^{1}\right)$ Cf. [Je03, Theorem 21.16]. Even worse, in some cases, even the consistency strength of $Z F C+L$ and $Z F+L$ is different. For instance, let $L$ be "there is a cardinal $\kappa$ such that $\mathcal{P}_{\kappa}\left(\kappa^{+}\right)$carries a normal measure". Then the consistency strength of ZF $+\mathrm{L}$ is bounded from above by $\omega$ Woodin cardinals ([Be81] in combination with Woodin's result on the consistency strength of AD; cf. [Ka03, Theorem 32.16]), whereas the consistency strength of ZFC $+\mathrm{L}$ is far bigger. (The last statement combines a result of Solovay on the failure of $\square_{\kappa}$ at a $\kappa^{+}$-supercompact cardinal $\kappa$ with inner model results by Schimmerling and Zeman [ScZe01]. For more details, see [Bo02, Kapitel 6].) 
In this paper, we investigate a large cardinal notion that is in conflict with negations of the axiom of choice: the notion of strong inaccessibility. A strongly inaccessible cardinal $\kappa$ is a regular strong limit cardinal where the traditional ZFC-definition of "strong limit" is that for all $\lambda<\kappa$, we have $2^{\lambda}<\kappa$.

In the ZF-context, this does not necessarily make sense, as $2^{\lambda}$ may not be well-ordered. We look at four different definitions of inaccessible cardinals that are equivalent in ZFC but not equivalent in ZF ( $\boldsymbol{i}$-inaccessibility, $\boldsymbol{v}$-inaccessibility, $\overline{\boldsymbol{s}}$-inaccessibility, and $\overline{\boldsymbol{z}}$-inaccessibility) and study the implicational structure of these notions (in ZF).

The paper is organized as follows: In $\S 2$, we present the definitions and their simple consequences. The four notions mentioned are presented in an implication diagram in Figure 1. In $\S 3$, we first finish the proof of the positive part of the implication diagram. After that, in $\S 4$, we briefly digress and discuss a property $\left(*_{\omega_{1}, \bar{\imath}}\right)$ that corresponds to being inaccessible without being a limit cardinal. The main part of the paper $(\S \S 5,6$, and 7$)$ contains the three independence results proving that none of the arrows in Figure 1 is reversible. Throughout this paper, the ambient theory is ZF.

2. Definitions of strong limits. Ordinarily, in set theory with the axiom of choice, we write $X \leq Y$ to mean that there is an injection from $X$ to $Y$, or equivalently (provided $X \neq \emptyset$ ) that there is a surjection from $Y$ to $X$. Without the axiom of choice, these two statements are not equivalent anymore and we have to be much more precise. We define four different notions of "being smaller than" for the context of ZF:

Let $X$ and $Y$ be nonempty sets and $\kappa$ be a cardinal. Then we write

- $X<_{i} \kappa$ if there is some $\alpha<\kappa$ and an injection from $X$ into $\alpha$,

- $X<_{s} \kappa$ if there is some $\alpha<\kappa$ and a surjection from $\alpha$ onto $X$,

- $X<_{\bar{\imath}} Y$ if there is no injection from $Y$ into $X$, and

- $X<_{\bar{s}} Y$ if there is no surjection from $X$ onto $Y$.

Of course, if $X$ and $Y$ are well-orderable, these four relations coincide. The following are immediate observations:

ObSERVATION 1. For a nonempty set $X$ and a cardinal $\kappa$, the statements $X<_{\boldsymbol{s}} \kappa$ and $X<_{\boldsymbol{i}} \kappa$ are equivalent and both imply that $X$ is well-orderable.

ObSERVATION 2. If $\kappa$ is a cardinal and $X$ is a nonempty set, then $X<_{s} \kappa$ implies $X<_{\bar{s}} \kappa$.

Observation 3. For nonempty sets $X$ and $Y$, if $X<_{\bar{s}} Y$, then $X<_{\bar{\imath}} Y$.

In the following, we shall use the symbol $\mathbf{x}$ as a placeholder for one of the four symbols $\boldsymbol{i}, \boldsymbol{s}, \overline{\boldsymbol{\imath}}$ and $\overline{\boldsymbol{s}}$. We say that a limit cardinal $\kappa$ is an $\mathbf{x}$-strong limit 
if for all $\lambda<\kappa$, we have $\mathcal{P}(\lambda)<_{\mathbf{x}} \kappa$, and we call an uncountable cardinal $\kappa$ $\mathbf{x}$-inaccessible if it is a regular $\mathbf{x}$-strong limit cardinal $\left({ }^{2}\right)$.

Of course, inaccessibility of a cardinal $\kappa$ is typically connected with metamathematical properties of $\mathbf{V}_{\kappa}$. This suggests another natural definition of inaccessibility: An ordinal $\kappa$ is $\boldsymbol{v}$-inaccessible if $\mathbf{V}_{\kappa}$ is a model of secondorder ZF. Here, by $\mathbf{V}_{\kappa}$ we mean the collection of sets of (Mirimanoff) rank $<\kappa$, regarded as a structure by interpreting $\in$ as actual membership. By "second-order ZF", we mean ZF with replacement strengthened to say that the image of a set under any function (not just under definable functions) is a set. It is easy to check the following characterizations of $\boldsymbol{v}$-inaccessibility.

Proposition 4. The following are equivalent, for any ordinal $\kappa$ :

(1) $\kappa$ is $\boldsymbol{v}$-inaccessible,

(2) $\kappa$ is a regular, uncountable cardinal and for all $\alpha<\kappa$, we have $\mathbf{V}_{\alpha}<_{\bar{s}} \kappa$

(3) $\kappa$ is a regular, uncountable cardinal and for all $\alpha<\kappa$ and all cofinal subsets $C \subseteq \kappa$, we have $\mathbf{V}_{\alpha}<_{\bar{s}} C$.

Combining Proposition 4 with the fact that $\mathcal{P}(\lambda) \subseteq \mathbf{V}_{\lambda+1}$, we immediately get the following connection between $\boldsymbol{v}$-inaccessibility and $\overline{\boldsymbol{s}}$-inaccessibility:

Corollary 5. Every $\boldsymbol{v}$-inaccessible cardinal is $\overline{\boldsymbol{s}}$-inaccessible.

The implication diagram in Figure 1 gives the connections between the five notions of inaccessibility. All implications except the ones labelled $(\ddagger)$ follow from the above simple observations and Corollary 5. The implication ( ) will be proved as Theorem 11 .

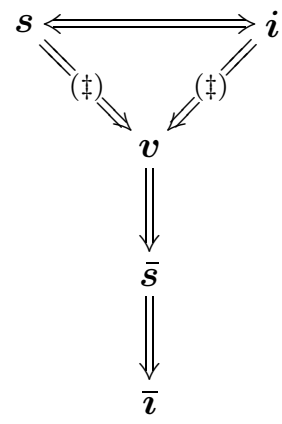

Fig. 1. The implication diagram

It is quite easy to see that the notion of an $\boldsymbol{i}$-strong limit is too strong for purposes of set theory without the axiom of choice:

$\left({ }^{2}\right)$ Here and for the rest of the paper, "cardinal" means "initial ordinal". 
Proposition 6. If there is an uncountable $\boldsymbol{i}$-strong limit, then there is a well-ordering of the real numbers.

Proof. If $\kappa$ is uncountable, then $\aleph_{0}<\kappa$, so by Observation $1, \mathcal{P}(\omega)$ is well-orderable.

We now use the notation IC for the assertion "there is an inaccessible cardinal" (meaning "there is an $\boldsymbol{i}$-inaccessible cardinal" if we use it in the $\neg \mathrm{AC}$-context). For the separating instances (without AC), we use the following notation:

- IC $\mathrm{I}_{\boldsymbol{v} / \neg \boldsymbol{i}}$ "there is an $\boldsymbol{v}$-inaccessible cardinal which is not $\boldsymbol{i}$-inaccessible",

- $\mathrm{IC}_{\overline{\boldsymbol{s}} / \neg \boldsymbol{v}}$ "there is an $\overline{\boldsymbol{s}}$-inaccessible cardinal which is not $\boldsymbol{v}$-inaccessible",

- $\mathrm{IC}_{\overline{\boldsymbol{\imath}} / \neg \overline{\boldsymbol{s}}}$ "there is an $\overline{\boldsymbol{\imath}}$-inaccessible cardinal which is not $\overline{\boldsymbol{s}}$-inaccessible".

The goal of this paper is to show that the arrows in the above diagram do not reverse in $\mathrm{ZF}$, i.e., that $\mathrm{ZF}+\mathrm{IC} \boldsymbol{c}_{\boldsymbol{v} / \neg \boldsymbol{i}}, \mathrm{ZF}+\mathrm{IC}_{\overline{\boldsymbol{s}} / \neg \boldsymbol{v}}$, and $\mathrm{ZF}+\mathrm{IC}_{\overline{\boldsymbol{\imath}} / \neg \overline{\boldsymbol{s}}}$ are consistent (cf. Theorems 17, 20, and 25). On the metamathematical side, it is interesting to note that the different definitions of strong inaccessibility do not give different consistency strength:

Proposition 7. If $\kappa$ is $\overline{\boldsymbol{\imath}}$-inaccessible, then $\kappa$ is inaccessible in $\mathbf{L}$.

Proof. Clearly, $\kappa$ is regular in $\mathbf{L}$. Let $\alpha<\kappa$. If $\mathbf{L} \models$ "there is an injection from $\kappa$ into $\mathcal{P}(\alpha)$ ", then there is an injection from $\kappa$ into $\mathcal{P}^{\mathbf{L}}(\alpha) \subseteq \mathcal{P}(\alpha)$, so $\kappa$ is not $\overline{\boldsymbol{z}}$-inaccessible in the universe.

COROLlary 8. The following are equiconsistent:

(1) ZFC + IC,

(2) ZF + "there is an $\boldsymbol{i}$-inaccessible",

(3) ZF + "there is an $\boldsymbol{v}$-inaccessible",

(4) ZF + "there is an $\bar{s}$-inaccessible",

(5) ZF + "there is an $\overline{\boldsymbol{\imath}}$-inaccessible".

The project of this paper was in part motivated by the behaviour of cardinals under the assumption of $Z F+A D$ where $A D$ denotes the axiom of determinacy (cf. [Ka03, § 27]) $\left(^{3}\right)$.

In the ZFC-context, the property $\left(*_{\kappa, \mathbf{x}}\right) \quad$ for all $\lambda<\kappa$, we have $\mathcal{P}(\lambda)<_{\mathbf{x}} \kappa$ can only hold for limit cardinals. This remains true without the axiom of choice for $<_{\bar{s}}$ :

$\left({ }^{3}\right)$ For an alternative definition of strong inaccessibility designed specifically for the AD-context, see [Ke85]. There, a cardinal $\kappa$ is strongly inaccessible if it is regular and for all $\lambda<\kappa$ there is a bijection between the set of ultrafilters on $\lambda$ and a proper initial segment of $\kappa$. Kechris proved (under the assumption of $A D+\mathbf{V}=\mathbf{L}(\mathbb{R})$ ) that $\boldsymbol{\delta}_{1}^{2}$ is strongly inaccessible in this sense. 
OBSERVATION 9. The statement $\left(*_{\left.\kappa^{+}, \bar{s}\right)}\right.$ is inconsistent with ZF.

Proof. In ZF, there is a surjection from $\mathcal{P}(\kappa)$ onto $\kappa^{+}$.

The analogue of Observation 9 for $\overline{\boldsymbol{\imath}}$ is not true: By a theorem of $\mathrm{My}-$ cielski's, AD implies that there cannot be an injection from $\omega_{1}$ into the reals [Ka03, Proposition 27.11(a)]; therefore $\left(*_{\omega_{1}, \bar{\imath}}\right)$ holds under AD. We will

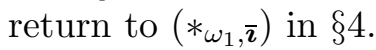

3. $\boldsymbol{v}$-inaccessible cardinals. In this section, we shall show the remaining implication $(\ddagger)$ from our diagram of implications. We start with a simple fact:

LEMMA 10. Let $\kappa$ be $\boldsymbol{i}$-inaccessible. If $\alpha<\kappa$ and $\mathbf{V}_{\alpha}$ is well-orderable, then $\mathbf{V}_{\alpha}<_{i} \kappa$.

Proof. Suppose not, and let $\alpha$ be the first counterexample. By $\boldsymbol{i}$-inaccessibility of $\kappa$, this $\alpha$ cannot be a successor ordinal, and it obviously is not 0 , so it is a limit ordinal. By minimality of $\alpha$, we have $\mathbf{V}_{\beta}<_{\boldsymbol{i}} \kappa$ for all $\beta<\alpha$. By regularity of $\kappa$, find a cardinal $\lambda<\kappa$ such that for all $\beta<\alpha$, we have $\mathbf{V}_{\beta}<_{\boldsymbol{i}} \lambda$.

Use the hypothesis to fix a well-ordering of $\mathbf{V}_{\alpha}$; it restricts to wellorderings of $\mathbf{V}_{\beta}$ for all $\beta<\alpha$, which then determine order-isomorphisms $f_{\beta}: \mathbf{V}_{\beta} \rightarrow \tau_{\beta}$, where, by our choice of $\lambda$, we have $\tau_{\beta}<\lambda$ for all $\beta<\alpha$. Combining all these maps $f_{\beta}$, we get a one-to-one map $g: \mathbf{V}_{\alpha} \rightarrow \alpha \times \lambda$ defined by

$$
g(x):=\left\langle\varrho(x), f_{\varrho(x)+1}(x)\right\rangle,
$$

where $\varrho(x)$ is the Mirimanoff rank of $x$. Since $\alpha \times \lambda$ is well-orderable with order-type $<\kappa$, this finishes the proof.

THEOREM 11. Every $\boldsymbol{i}$-inaccessible cardinal is $\boldsymbol{v}$-inaccessible.

Proof. Let $\kappa$ be $\boldsymbol{i}$-inaccessible. By definition, $\kappa$ is regular and uncountable, so it remains to prove that no $\mathbf{V}_{\alpha}$ with $\alpha<\kappa$ can be mapped onto $\kappa$. In fact, we shall prove more, namely that every such $\mathbf{V}_{\alpha}$ is well-orderable and has cardinality $<\kappa$.

By Lemma 10, it remains to show that $\mathbf{V}_{\alpha}$ is well-orderable for all $\alpha<\kappa$. Suppose not, and let $\alpha$ be the smallest counterexample. So $\mathbf{V}_{\beta}$ is wellorderable and, by Lemma 10, of cardinality $<\kappa$ for all $\beta<\alpha$. As in the proof of Lemma 10, $\alpha$ must be a limit ordinal, and we can obtain a cardinal $\lambda<\kappa$ such that $\mathbf{V}_{\beta}<_{\boldsymbol{i}} \lambda$ for all $\beta<\alpha$. Thus, each such $\mathbf{V}_{\beta}$ has a well-ordering of length $<\lambda\left({ }^{4}\right)$.

$\left({ }^{4}\right)$ If we could choose one such well-ordering for each $\beta<\alpha$, then we could combine these, as in the proof of the lemma, to well-order $\mathbf{V}_{\alpha}$. Unfortunately, we cannot immediately make such choices. The rest of the proof is devoted to getting such choices without invoking the axiom of choice. 
By $\boldsymbol{i}$-inaccessibility of $\kappa$, fix a well-ordering $\prec$ of $\mathcal{P}(\lambda)$. We inductively define, for $\beta \leq \alpha$, specific well-orderings $\prec_{\beta}$ of $\mathbf{V}_{\beta}$ as described below. Note that the induction includes $\beta=\alpha$, so we will obtain a well-ordering $\prec_{\alpha}$ of $\mathbf{V}_{\alpha}$, thereby completing the proof.

Let $\prec_{0}$ be the empty relation (on the empty set $\mathbf{V}_{0}=\emptyset$ ). For limit $\beta$, let

$$
x \prec_{\beta} y: \Leftrightarrow \varrho(x)<\varrho(y) \text { or }\left(\varrho(x)=\varrho(y)=\gamma \text { and } x \prec_{\gamma+1} y\right) .
$$

Finally, for a successor ordinal $\beta=\gamma+1$, notice that the order-isomorphism from $\prec_{\gamma}$ to its order-type $\tau$ maps $\mathbf{V}_{\gamma}$ one-to-one into $\lambda$ (as our choice of $\lambda$ ensures $\tau<\lambda)$. This induces a one-to-one map of $\mathbf{V}_{\beta}=\mathcal{P}\left(\mathbf{V}_{\gamma}\right)$ into $\mathcal{P}(\lambda)$. Use this map to pull back the fixed well-ordering of $\mathcal{P}(\lambda)$ to a well-ordering of $\mathbf{V}_{\beta}$, and define that well-ordering to be $\prec \beta$.

4. The property $\left(*_{\omega_{1}, \overline{\boldsymbol{\imath}}}\right)$. In this section, we briefly return to the property $\left(*_{\kappa, \mathbf{x}}\right)$ for successor cardinals $\kappa$. We will discuss the case $\kappa=\omega_{1}$. We have seen that $\left(*_{\omega_{1}, \overline{\boldsymbol{s}}}\right)$ is refutable in ZF, but $\left(*_{\omega_{1}, \overline{\mathbf{v}}}\right)$ is a theorem of ZF+AD. Even more, ZF $+\left(*_{\omega_{1}, \bar{\imath}}\right)$ is equiconsistent with ZF. For this, we give the following simple argument using the Feferman-Lévy model which is a folklore result well-known in the community:

The Feferman-Lévy model (cf. [FeLé63] and [Je03, Example 15.57]) is a symmetric submodel of a generic extension that satisfies " $\mathbb{R}$ is a countable union of countable sets" ( $\neg 38$ in the notation of [HoRu98]).

Lemma 12. If $X$ is a countable union of countable sets, then there is no surjection from $X$ onto $\aleph_{1}^{\aleph_{0}}$.

Proof. This is essentially the diagonal argument for König's Theorem: Let $X=\bigcup_{n \in \omega} X_{n}$ for some countable sets $X_{n}$ and let $\pi$ be any function from $X$ to $\aleph_{1}^{\aleph_{0}}$. Then $\pi\left[X_{n}\right]$ is a countable set, and therefore $\omega_{1} \backslash\left\{\pi(x)(n) ; x \in X_{n}\right\}$ $\neq \emptyset$. Let $\alpha_{n}$ be the least element of this set. Define $Z: \omega \rightarrow \omega_{1}$ by $Z(n):=$ $\alpha_{n}$. Then $Z$ is not in the range of $\pi$.

LEMMA 13. If there is an injection from $\omega_{1}$ into the reals $(170$ in the notation of [HoRu98]), then there is a bijection between the reals and $\aleph_{1}^{\aleph_{0}}$.

Proof. By the assumption, we have an injection from $\aleph_{1}$ into $2^{\aleph_{0}}$, and hence from $\aleph_{1}^{\aleph_{0}}$ into $\left(2^{\aleph_{0}}\right)^{\aleph_{0}}$ and thus into $2^{\aleph_{0}}$. By the Schröder-Bernstein theorem, we get a bijection from $2^{\aleph_{0}}$ to $\aleph_{1}^{\aleph_{0}}$.

Obviously, Lemmas 12 and 13 show that the Feferman-Lévy model satisfies $\left(* \omega_{1}, \overline{\mathbf{z}}\right)$.

Corollary 14. If Cons(ZFC), then $\operatorname{Cons}\left(\mathrm{ZF}+\left(*_{\omega_{1}, \bar{\imath}}\right)\right)$. 
5. Cardinals that are not $\boldsymbol{i}$-inaccessible. For the remainder of the paper, we shall freely use the notation for forcing and symmetric submodels of generic extensions as laid out in [Je03, pp. 249-261]. In the ZFC context, we can recursively define for any cardinal $\mu$ its beth sequence by $\beth_{0}(\mu):=\mu$, $\beth_{\alpha+1}(\mu):=2^{\beth_{\alpha}(\mu)}$, and $\beth_{\delta}(\mu):=\bigcup\left\{\beth_{\alpha}(\mu) ; \alpha<\delta\right\}$ (for limit ordinals $\delta$ ).

LEMma 15. Let $M$ be a model of ZFC. Assume that, in $M, \lambda, \mu$, and $\kappa$ are cardinals satisfying $2^{\lambda}<\kappa, 2^{\mu}<\kappa$. Assume further that, in $M$, $\mathbb{P}$ is a partial order with $\operatorname{Card}(\mathbb{P})=\mu$. Let $G$ be $\mathbb{P}$-generic over $M$, and $M \subseteq N \subseteq M[G]$ such that $N$ is a model of ZF. Then

$$
N \models 2^{\lambda}<_{\bar{s}} \kappa .
$$

Similarly, if in addition, in $M, \beth_{\lambda}(\mu)<\kappa$, then

$$
N \models \mathbf{V}_{\lambda}<_{\bar{s}} \kappa \text {. }
$$

Proof. This lemma is a standard and basic technique of forcing. Let us call a $\mathbb{P}$-name $\tau$ canonical if it consists of pairs $\langle\check{\alpha}, p\rangle$ for $\alpha<\lambda$. Obviously, there are at most $\left(2^{\operatorname{Card}(\lambda \times \mathbb{P})}\right)^{M}$ many canonical names. In $M[G]$, every subset of $\lambda$ has a canonical name, so we have

$$
\left(2^{\lambda}\right)^{M[G]} \leq\left(2^{\operatorname{Card}(\lambda \times \mathbb{P})}\right)^{M}=\left(2^{\max \{\lambda, \mu\}}\right)^{M}<\kappa .
$$

Therefore, $M[G] \models$ "there is no surjection from $2^{\lambda}$ onto $\kappa$ ".

Suppose, towards a contradiction, $N$ has a surjection from $\left(2^{\lambda}\right)^{N}$ onto $\kappa$. This persists in $M[G]$ and (since $\left(2^{\lambda}\right)^{N} \subseteq\left(2^{\lambda}\right)^{M[G]}$ ) can be trivially extended to a surjection from $2^{\lambda}$ onto $\kappa$ in $M[G]$. Contradiction!

For the additional claim, we define canonical $\alpha$-names for elements of $\mathbf{V}_{\alpha}$ by recursion: Elements $F$ of $\mathbf{V}_{\omega}$ are absolute and can be represented by the name $\breve{F}$. These names are the canonical 0-names. If $C$ is the set of canonical $\alpha$-names (for elements of $\mathbf{V}_{\omega+\alpha}$ ), then a canonical $\alpha+1$-name is a subset of $C \times \mathbb{P}$. Recursively, it is easy to see that there are at most $\beth_{\lambda}(\mu)^{M}$ many canonical $\lambda$-names, so $M[G] \models$ "there is no surjection from $\mathbf{V}_{\lambda}$ onto $\kappa$ ", which again persists downwards to $N$.

Corollary 16. Let $M$ be a model of ZFC + IC and let $\kappa \in M$ be inaccessible. If $\mathbb{P} \in M$ is a partial order with $M \models \operatorname{Card}(\mathbb{P})<\kappa$ and $N$ is a ZF-model such that $M \subseteq N \subseteq M[G]$, then

$$
N \models \text { " } \kappa \text { is } \boldsymbol{v} \text {-inaccessible". }
$$

Proof. Since $\operatorname{Card}(\mathbb{P})<\kappa, \kappa$ remains regular in $M[G]$ and hence in $N$. The rest follows from Lemma 15.

Theorem 17. If Cons $(\mathrm{ZFC}+\mathrm{IC})$, then $\operatorname{Cons}\left(\mathrm{ZF}+\mathrm{IC}_{\boldsymbol{v} / \neg \boldsymbol{i}}\right)$.

Proof. Assume that $\kappa$ is strongly inaccessible in $M \models Z F C$. If $\mathbb{P}$ is Cohen forcing and $G$ is $\mathbb{P}$-generic over $M$, we can create a symmetric submodel $N \subseteq M[G]$ such that the reals in $N$ are not well-ordered (cf. [Je03, Example 
15.52]). By Proposition 6 , we know that $N \models$ " $\kappa$ is not $\boldsymbol{i}$-inaccessible". But by Corollary $16, N \models$ " $\kappa$ is $\boldsymbol{v}$-inaccessible".

6. Cardinals that are not $v$-inaccessible. In this section, we will construct a model of $\mathrm{ZF}+\mathrm{IC}_{\overline{\boldsymbol{s}} / \neg \boldsymbol{v}}$. We start with a ground model $M$ satisfying ZFC+ "there exists an inaccessible" and let $\kappa$ be a fixed inaccessible cardinal in $M$. Consider the partial order

$$
\mathbb{P}:=\operatorname{Fn}(\kappa \times \omega, 2)
$$

adding a $\kappa$-sequence of Cohen reals. Let $\mathbf{G}$ be the group of all permutations of $\kappa$, acting on $\mathbb{P}$ by

$$
\pi(p)(\alpha, n):=p\left(\pi^{-1}(\alpha), n\right) ;
$$

it permutes the canonical names $\dot{c}_{\alpha}$ of the Cohen reals by acting on the subscripts $\alpha$. If $A \subseteq \kappa$ is a set, then let $K_{A} \subseteq \mathbf{G}$ be the set of those permutations that fix the elements of $A$. Now take the filter $\mathcal{F}$ on $\mathbf{G}$ generated by the set $\left\{K_{A} ; A \subseteq \kappa, \operatorname{Card}(A)<\kappa\right\}$. Fix a $\mathbb{P}$-generic filter $G$ over $M$ and let $N$ be the symmetric submodel of $M[G]$ defined by $\mathcal{F}$.

Clearly, $c_{\alpha} \in N$, as $K_{\{\alpha\}}$ is a support of its canonical name. For each $\lambda<\kappa$, the initial segment $C_{\lambda}:=\left\langle c_{\alpha} ; \alpha<\lambda\right\rangle$ of the adjoined $\kappa$-sequence of Cohen reals is in $N$, because its canonical name is supported by $K_{\lambda}$.

Proposition 18. In $N$, there is a surjection from $\mathbf{V}_{\omega+4}$ onto $\kappa$; in particular, $\kappa$ is not $\boldsymbol{v}$-inaccessible (by Lemma 4 ).

Proof. Consider, in $N$, the function $t$ assigning to every well-ordering (in $N$ ) of a subset (in $N$ ) of $\mathcal{P}^{N}(\omega)$ its order-type. For every $\lambda<\kappa$, the set $C_{\lambda} \in N$ witnesses that $\lambda \in \operatorname{ran}(t)$. But the domain of $t$ is a subset of $\mathbf{V}_{\omega+4}$.

Proposition 19. In $N$, $\kappa$ is $\overline{\boldsymbol{s}}$-inaccessible.

Proof. Consider $\lambda<\kappa$ and suppose, toward a contradiction, that $N$ contains a map of $\mathcal{P}^{N}(\lambda)$ onto $\kappa$. Fix, in the ground model $M$, a name $\dot{f}$ and a condition $p$ such that $p \Vdash " \dot{f}: \mathcal{P}^{N}(\lambda) \rightarrow \kappa$ is surjective".

For the rest of this proof, we shall work in $M$. Fix $\mu<\kappa$ large enough so that $K_{\mu}$ supports $\dot{f}$ and so that $\operatorname{dom}(p) \subseteq \mu \times \omega$.

Fix some $\alpha<\kappa$. Since $p$ forces $\alpha$ to be in the range of $\dot{f}$, we can choose a nice name $\dot{x}_{\alpha}$ for a subset of $\lambda$ and choose a condition $q_{\alpha}$ extending $p$ and forcing that $\dot{f}\left(\dot{x}_{\alpha}\right)=\alpha$. Thanks to the countable chain condition of $\mathbb{P}$, there is a set $I \subseteq \kappa$ such that $\operatorname{Card}(I) \leq \lambda, \operatorname{dom}\left(q_{\alpha}\right) \subseteq I \times \omega$, and $\operatorname{dom}(r) \subseteq I \times \omega$ for every condition $r$ involved in the nice name $\dot{x}_{\alpha}$. (Note that the conditions involved in a nice name for a subset of $\lambda$ constitute $\lambda$ many antichains, each of which is countable.) Let $\pi_{\alpha} \in \mathbf{G}$ be a permutation that fixes all ordinals 
$<\mu$ but moves all ordinals in $I \backslash \mu$ to ordinals between $\mu$ and $\mu+\lambda$ (ordinal sum). Then $\pi_{\alpha}$ fixes $\dot{f}$ and $p$, by our choice of $\mu$. Therefore:

(1) $\pi_{\alpha}\left(\dot{x}_{\alpha}\right)$ is a nice name for a subset of $\lambda$, and all conditions involved in it have domains $\subseteq(\mu+\lambda) \times \omega$.

(2) $\pi_{\alpha}\left(q_{\alpha}\right)$ is a condition extending $p$ and having domain $\subseteq(\mu+\lambda) \times \omega$.

(3) $\pi_{\alpha}\left(q_{\alpha}\right) \Vdash \dot{f}\left(\pi_{\alpha}\left(\dot{x}_{\alpha}\right)\right)=\alpha$.

Now let $\alpha$ vary over all ordinals $<\kappa$. The numbers of possibilities for $\pi_{\alpha}\left(\dot{x}_{\alpha}\right)$ and $\pi_{\alpha}\left(q_{\alpha}\right)$ are, thanks to the $\mu+\lambda$ bounds in (1) and (2), strictly smaller than $\kappa$, because $\kappa$ is inaccessible (in $M$ ). So there must be some $\alpha<\beta<\kappa$ with $\pi_{\alpha}\left(\dot{x}_{\alpha}\right)=\pi_{\beta}\left(\dot{x}_{\beta}\right)$ and $\pi_{\alpha}\left(q_{\alpha}\right)=\pi_{\beta}\left(q_{\beta}\right)$. But then (3) and the corresponding assertion for $\beta$ say that one and the same condition forces one and the same term to have two distinct values, a contradiction.

Theorem 20. If Cons $(\mathrm{ZFC}+\mathrm{IC})$, then $\operatorname{Cons}\left(\mathrm{ZF}+\mathrm{IC}_{\overline{\boldsymbol{s}} / \neg \boldsymbol{v}}\right)$.

7. Cardinals that are not $\bar{s}$-inaccessible. In this last section, we will construct a model of ZF $+\mathrm{IC}_{\overline{\mathbf{z}} / \neg \overline{\boldsymbol{s}}}$. We again start with a ground model $M$ satisfying ZFC+ "there exists an inaccessible" and let $\kappa$ be a fixed inaccessible cardinal in $M$. Here we consider the partial order

$$
\mathbb{P}:=\operatorname{Fn}(\kappa \times \omega \times \omega, 2)
$$

which adds for each $\alpha<\kappa$ a countable set of Cohen reals $\{c(\alpha, n) ; n \in \omega\}$. We are going to permute these sets so that we cannot pick from these countable sets.

Let $\mathbf{G}$ be the group of permutations of $\kappa \times \omega$ that fix the first component, i.e., the direct product of $\kappa$ copies of the group of all permutations of $\omega$. Let this group act on $\mathbb{P}$ by permuting the indices $\langle\alpha, n\rangle$ of the Cohen reals, i.e.,

$$
\pi(p)(\alpha, n, m):=p\left(\pi^{-1}(\alpha, n), m\right) .
$$

Similarly to $\S 6$, we let $K_{A} \subseteq \mathbf{G}$ be the set of those permutations that fix the elements of $A \subseteq \kappa \times \omega$. This time, we take the filter $\mathcal{F}$ on $\mathbf{G}$ generated by the set $\left\{K_{A} ; A \subseteq \kappa \times \omega\right.$ finite $\}$. Fix a $\mathbb{P}$-generic filter $G$ over $M$ and let $N$ be the symmetric submodel of $M[G]$ defined by $\mathcal{F}$. Clearly, $c(\alpha, n) \in N$, as $K_{\{\langle\alpha, n\rangle\}}$ is a support of its canonical name.

Lemma 21. In $N$, the cardinal $\kappa$ is not $\bar{s}$-inaccessible.

Proof. It is enough to show that there is a surjection from the reals onto $\kappa$ in $N$. We consider the function $f \in M[G]$ defined by

$$
f(x):= \begin{cases}\alpha & \text { if } x=c(\alpha, n) \text { for some } n, \\ 0 & \text { otherwise. }\end{cases}
$$

It is easy to see that $f$ has a hereditarily symmetric name, and thus $f \in N$. 
We now turn to the proof that $\kappa$ is $\overline{\boldsymbol{\imath}}$-inaccessible in $N$. For $A \subseteq \kappa \times \omega$, $p \in \mathbb{P}$ and $J \subseteq \mathbb{P}$, we define

$$
\begin{aligned}
& p^{A}:=\{\langle\alpha, n, m, i\rangle \in p ;\langle\alpha, n\rangle \in A\}=p \cap(A \times \omega \times 2), \\
& J^{A}:=\left\{p^{A} ; p \in J\right\} .
\end{aligned}
$$

Lemma 22. Fix some finite $A \subseteq \kappa \times \omega$. Let $\varphi$ be a formula of the forcing language such that for all names $\tau$ occurring in $\varphi$, we have $K_{A} \subseteq \operatorname{sym}(\tau)$. Then the following holds for all $p \in \mathbb{P}$ :

$$
\text { If } p \Vdash \varphi \text {, then } p^{A} \Vdash \varphi \text {. }
$$

Proof. If $p^{A} \nVdash \varphi$, then there is a $q \supseteq p^{A}$ such that $q \Vdash \neg \varphi$. Find a permutation $\pi \in K_{A}$ such that $\pi(p)$ and $q$ are compatible, i.e., there is an $r$ such that $r \leq \pi(p)$ and $r \leq q$. Then $r \Vdash \neg \varphi$. Clearly, $\pi(p)$ forces $\varphi^{\pi}$, the formula obtained from $\varphi$ by replacing all names occurring in $\varphi$ by their $\pi$-images. Since $\pi \in K_{A}$, and by assumption all of the names in $\varphi$ are supported by $K_{A}$, we get $\varphi^{\pi}=\varphi$, so $r \Vdash \varphi$, which is absurd.

We shall now look at intermediate models between $M$ and $M[G]$. For some finite $A \subseteq \kappa \times \omega$, we consider the forcing notion $\mathbb{P}^{A}=\left\{p^{A} ; p \in \mathbb{P}\right\}$ which is forcing-equivalent to ordinary Cohen forcing. Clearly, $G^{A}=\left\{p^{A}\right.$; $p \in G\}$ is a $\mathbb{P}^{A}$-generic filter over $M$, and $M\left[G^{A}\right]$ is essentially the Cohen model.

Lemma 23. If $X \in N$ is a set of ordinals, then there is a finite $A \subseteq \kappa \times \omega$ such that $X \in M\left[G^{A}\right]$.

Proof. Since $X \in N$, it has a hereditarily symmetric $\mathbb{P}$-name $\dot{X}$ (with respect to $G)$. Fix a finite set $A \subseteq \kappa \times \omega$ such that $K_{A} \subseteq \operatorname{sym}(\dot{X})$. Let $\varphi_{\alpha} \bumpeq \check{\alpha} \in \dot{X}$. Clearly, for each $\alpha$ there is some $p_{\alpha} \in G$ such that $p_{\alpha} \Vdash \varphi_{\alpha}$ or $p_{\alpha} \Vdash \neg \varphi_{\alpha}$. For every ordinal $\alpha$, the formula $\varphi_{\alpha}$ satisfies the requirement of Lemma 22. Therefore, $p_{\alpha}^{A} \Vdash \varphi_{\alpha}$ if and only if $p_{\alpha} \Vdash \varphi_{\alpha}$, and hence $X \in$ $M\left[G^{A}\right]$.

TheOREM 24. In $N$, the cardinal $\kappa$ is $\overline{\boldsymbol{\imath}}$-inaccessible.

Proof. Since our partial order has the c.c.c., it preserves cofinalities. Therefore $\kappa$ is regular in $M[G]$ and hence still regular in $N$. It remains to show that $\kappa$ cannot be embedded in any $2^{\lambda}$ for $\lambda<\kappa$.

Assume towards a contradiction that there is in $N$ an injection $h: \kappa \rightarrow 2^{\lambda}$ for some $\lambda<\kappa$. Such an injection can be viewed as a subset of $\kappa \times \lambda$, and therefore as a set of ordinals. We apply Lemma 23 to $h$ and deduce that there is some finite $A \subseteq \kappa \times \omega$ such that $h \in M\left[G^{A}\right]$ and thus

$$
M\left[G^{A}\right] \models \kappa \leq 2^{\lambda} .
$$

Keeping in mind that $\operatorname{Card}\left(\mathbb{P}^{A}\right)=\aleph_{0}$, we can apply the proof of Lemma 15 to find that $M \models \kappa \leq 2^{\lambda}$, which is false. 


\section{Theorem 25. If Cons $(\mathrm{ZFC}+\mathrm{IC})$, then $\operatorname{Cons}\left(\mathrm{ZF}+\mathrm{IC}_{\overline{\boldsymbol{\imath}} / \neg \overline{\boldsymbol{s}}}\right)$.}

\section{References}

[Be81] H. S. Becker, AD and the supercompactness of $\aleph_{1}$, J. Symbolic Logic 46 (1981), 822-842.

[Bo02] S. Bold, $A D$ und Superkompaktheit, Diplomarbeit, Rheinische FriedrichWilhelms-Universität Bonn, 2002.

[FeLé63] S. Feferman and A. Lévy, Independence results in set theory by Cohen's method II, Notices Amer. Math. Soc. 10 (1963), 593 (abstract).

[HoRu98] P. Howard and J. E. Rubin, Consequences of the Axiom of Choice, Math. Surveys Monogr. 59, Amer. Math. Soc., 1998.

[Je03] Th. Jech, Set Theory, 3rd ed., Springer Monogr. Math., Springer, 2003.

[Ka03] A. Kanamori, The Higher Infinite, Springer Monogr. Math., Springer, 2003.

[Ke85] A. S. Kechris, Determinacy and the structure of $\mathbf{L}(\mathbb{R})$, in: Recursion Theory (Ithaca, NY, 1982), A. Nerode and R. A. Shore (eds.), Proc. Sympos. Pure Math. 42, Amer. Math. Soc., 1985, 271-283.

[ScZe01] E. Schimmerling and M. Zeman, Square in core models, Bull. Symbolic Logic 7 (2001), 305-314.

Department of Mathematics

University of Michigan

Ann Arbor, MI 48109-1043, U.S.A.

E-mail: ablass@umich.edu

Mathematisches Institut

Rheinische Friedrich-Wilhelms-Universität Bonn

Beringstrasse 1

53115 Bonn, Germany

E-mail: dimitri@math.uni-bonn.de
Institute for Logic, Language and Computation

Universiteit van Amsterdam Plantage Muidergracht 24 1018 TV Amsterdam, The Netherlands E-mail: bloewe@science.uva.nl

Department Mathematik Universität Hamburg Bundesstrasse 55 20146 Hamburg, Germany

Received 29 July 2006;

in revised form 23 November 2006 